\title{
An Electricity Day-Ahead Market Simulation Model
}

\author{
T. Nogueira ${ }^{1}$, A. Vale ${ }^{2}$ and Z. Vale ${ }^{3}$ \\ ${ }^{1,3}$ Department of Electrical Engineering \\ Insitute of Engineering, Polytechnique Institute of Porto \\ Rua Dr. António Bernardino de Almeida, 4200-072 Porto (Portugal) \\ phone:+351 228340 500, fax:+351228321 159, \\ e-mail: talex@dee.isep.ipp.pt, zav@dee.isep.ipp.pt \\ ${ }^{2}$ Department of Electrical and Computer Engineering \\ Faculty of Engineering of University of Porto \\ Rua Dr. Roberto Frias, 4200-465 Porto (Portugal) \\ phone:+351225 081819, fax:+351225081 440, \\ e-mail: avale@fe.up.pt
}

\begin{abstract}
Several years have passed since the first efforts to restructure the electricity sector. Today many countries have already introduced electricity restructuring reform laws, a process commonly referred as deregulation. The arguments for deregulation include expected increased competition, new system efficiency, lower average costs to consumers and the promise of future improvement as markets grow in size and sophistication. The restructuring process has gone beyond the point of no return. In the European Union there are clear directives that impose the member states deadlines to implement competitive markets. Such is the case of Portugal and Spain, whose governments have decided to establish an Iberian electricity market.

This paper presents a simulation prototype of a competitive daily market, including pool and bilateral transactions, inspired on the system that has been successfully performing in Spain. The simulator provides users a means to obtain a deeper knowledge and awareness of a competitive electricity market.
\end{abstract}

\section{Key words}

Electricity markets, simulation model, power producers, deregulation, power pool.

\section{Introduction}

For the past few years many countries have undertaken the restructuring of the electricity sector. Following the first efforts pioneered by Chile, Argentina and England and Wales, several countries around the world, namely U.S. states and European Union state members, have introduced electricity restructuring reform laws, a process commonly referred as deregulation. Most experiences have been a qualified success, resulting in gains in terms of economic efficiency, but some have led to failure, the implemented market rules being unable to provide lower costs [1] or assure reliability and safety (California is an example).

The move toward an electricity market environment has the essential purpose of enhancing competition and inducing efficiency and lower electricity costs [2]. Although national experiences differ as regards trading rules, market operations, and the extent to which deregulation is carried out, the very same entities arise as performing a role in the new competitive framework: the regulator, the power producers, the selling agents and buyers, the market and system operators, and the consumers.

No matter what market model is adopted, electricity, seen as a good, should be separated from the services needed to deliver it to consumers. This perspective does not modify the physical flow of electricity from producers to consumers, but it certainly changes the way one sells, buys and trades electricity.

Abandoning the traditional vertically integrated power utilities - production, transmission and distribution activities within a single monopolist entity - the new organization of the markets foresees the splitting of the commercial and technical dimensions. New distinctive business areas emerge, with their own products and services.

The restructuring process, however, is strongly limited by the nature of the electricity activities - there are physical laws that differentiate the electricity business from others. Furthermore, there is a difficulty in adopting the principles of a free competitive market in a sector that favors the formation or maintenance of monopolies. The nature of electricity does not allow to abandon neither regulation, nor the need of a central coordination of the electrical system, remaining the activity of transmission a monopoly. The organization and the rules of the market must assure the conditions that, together with the regulatory aspects, lead to a competitive environment as much as possible, from which every player in the industry - consumers included - will eventually benefit.

Having recognized the new market environment, the question lies on how does one implement a structure that is able to assure a competitive framework, given a significant number of constraints, both of economic and 
technical nature.

As suggested by Flechner [3], one can consider the electricity sector as having two dimensions: a real dimension, that has to do with the physical flow of electricity, and a virtual dimension, related to the trading of electricity. Within the virtual dimension, buyers and sellers establish contracts, either physical contracts (which intend to provide for real electricity) or not physical contracts (designed to hedging risk through buying and selling futures and options). Both dimensions are intimately connected, but still a separation must be drawn in order to build an operational competitive environment.

The time dimension of the transactions in an open electricity market is of extreme importance. Either through a power exchange or a pool mechanism, or through bilateral contracts, electricity transactions can be made within different markets at the same time: spot markets, forward markets, future markets, swap markets and planning markets [4].

The simulator described in this paper addresses a market structure where forward bilateral contracts to deliver certain amounts of electricity for a particular day are known, as well as an auction mechanism that allows buyers and sellers to post their bids for that same day. An independent operator clears the market and produces a dispatch for that day.

\section{Day-ahead Market Organization}

\section{A. Electricity Market Organization}

In a competitive electricity market one can find an independent system operator (ISO), whose task is to keep the physical integrity of the transmission system while providing nondiscriminatory access to all participants in the market [5]. It is expected of the ISO to settle an optimal operation schedule at every time, given the physical constraints of the transmission lines. As in the Spanish electricity market, one can separate this mission into two distinct roles. A first role is that of a market operator, who must ensure that all participants are able to access the system and who should produce a feasible dispatch based on the information of price and quantity from sellers and buyers. A second role is that of a system operator, that must check the feasibility of the transactions, taking into account the physical transmission capacity and other technical constraints.

There are two main models for a market structure: the pool and the bilateral market. The pool is a market structure, with which suppliers and buyers transact. Some sort of auction is implemented [6], and, based on the quantities and prices offered by suppliers and buyers, a market operator should clear the market, dispatching the least expensive suppliers. In a bilateral market, sellers and buyers transact directly with each other, which implies that the participants should look themselves for information about prices.

There are arguments in favor of the pool system, and in favor of the bilateral system. Nevertheless, both systems can exist at the same time, provided that the ISO ensures the necessary coordination. This combined system thus implements an auction through which suppliers submit their production bids and buyers submit their demand bids. A certain price will clear the market and a certain amount of electricity is to be accepted for production and consumption. The bilateral contracts are then taken into account, and those that are feasible will be part of the dispatch. This mechanism must be performed on a regular basis (hourly auctions are frequent) and adjustments are often necessary to ensure that the dispatch is feasible.

\section{B. Day-ahead Market}

In real time there must be a completely centralized coordination, as supply must equal demand at every second. This coordination has to balance the system continuously, at all times [7].

In many real markets there is a day-ahead market (DA). Most transactions are cleared in the DA market for each hour of the next day. The risk of the participants of the market, who can see the spot price change significantly along the day, can be managed through contracts for differences (CFD), which cover the differences between the price of the transactions in the DA market and the spot prices in real time (that is, on the next day, when the physical transaction is to take place).

The Spanish system relies on such a market structure. There is an organized part that includes the electricity market (a daily and an intra-daily market) and market for ancillary services, and a non-organized part, to carry out bilateral transactions. In the day-ahead market, suppliers submit their selling bids and prices for each hour of the day, using an e-commerce marketplace, and buyers submit their demand bids as well. These bids are processed using a matching algorithm: the selling bids are ordered by increasing prices, and the demand bids are ordered by decreasing prices, resulting in an aggregated supply curve and an aggregated demand curve. The intersection of these curves determines the market clearing price (that of the last accepted selling bid). The market operator (MO) then broadcasts the dispatch, which states the matching bids. The system operator works closely with the market operator to determine the electricity transactions to take place and the commitment of the generators needed to carry out the economic transactions.

The sellers are allowed to include some degree of complexity in their bids. In fact, there might be constraints of indivisibility (thermal units frequently have such constraints, as they face significant start-up costs). Also, the first quantity a generator bids is always indivisible, which means that if a bid is accepted, it should be for the total quantity and not for just a part of it. There might be a minimum up-time, as well: once a thermal unit is on, it should be committed for a certain amount of time. These conditions are placed by the seller together with the price/quantity bids. 
After the market matching is done, the system operator analyses the physical feasibility of the market operator dispatch. If necessary, ancillary services are used and corrections are introduced. As a result, in the day-ahead market every transaction for each hour of the next day is programmed. From then on, as time goes by, an intra-daily market allows for adjustments.

\section{A Day-ahead Market Simulation Model}

This paper presents a market simulation model that we have developed to provide deeper knowledge of the operations of a day-ahead wholesale market. Figure 1 shows the flow of operations that have been implemented.

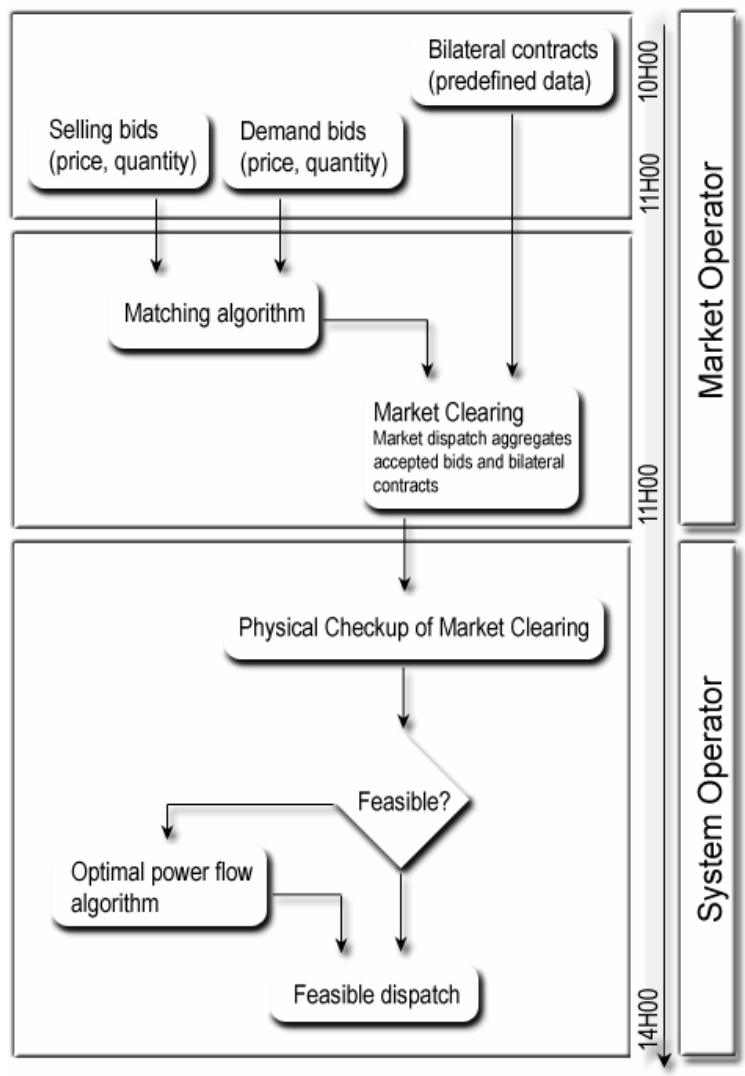

Fig. 1. Flow of operations implemented in the simulation process.

In order to permit participants to interact simultaneously, a standard web-based interface has been chosen. The simulator has been written in VB Script language and uses Active Server Pages technology.

The user interacts with a browser and participates assuming the role of a seller, a buyer or a ISO. Both sellers and buyers are requested to post their bids, but neither will have access to information other than historic data and results of the market operator's dispatch. One participant only can assume the ISO's role, gaining access to all data, including, of course, the bids that are posted in the DA market and the bilateral contracts previously placed for the next day. The ISO player will not interfere with the bidding process, except when he or she decides to change the parameters of the simulation itself (for example, the rules of generator's bidding).

The information posted by bidders, as well as the data that defines the electrical network, the transmission lines and the generators characteristics, are registered in a Microsoft Access database. This database also contains predefined historic time series data of bilateral contracts, which are used to set bilateral transactions of electricity for the next day. So far, users cannot interfere with this predefined set of bilateral contracts, but the simulation can be set up to randomly generate other sets of bilateral contracts, according to a selectable probabilistic distribution.

Generators are allowed to produce up to five-block bids for every hour. The simulation can be set up to accept the extra condition not to divide the first block bid of a generator. An imperative condition for a seller to participate in the auction is to produce at least one block bid for every hour.

Buyers also place their demand bids. These can be price/quantity bids or simple quantity bids (in this case the simulator assumes the bidder is paying the maximum price allowed for each unit of energy, which is a parameter that can be set in the simulation).

Once the bids are posted, the market operator checks whether any generator that bids is violating its operational constraints. Then he activates a matching algorithm that considers all bids to be simple. The results are then corrected to comply with complex conditions (for example, the market operator verifies the indivisible first block condition) and the market clearing is produced. Figure 2 depicts the matching algorithm that follows the steps previously described, and shows a result obtained in a simulation experiment.

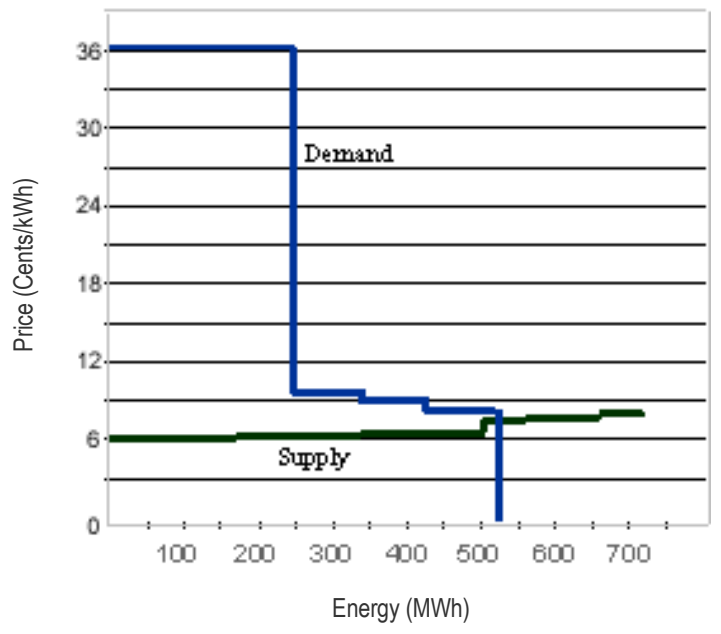

Fig. 2. Results of the matching algorithm for the $8^{\text {th }}$ hour of the next day in a simulation experiment.

As can be seen in figure 2, the first segment of the demand curve for the $8^{\text {th }}$ hour refers to buyer simple quantity bids at the maximum price of $0,36 € / \mathrm{MWh}$. 


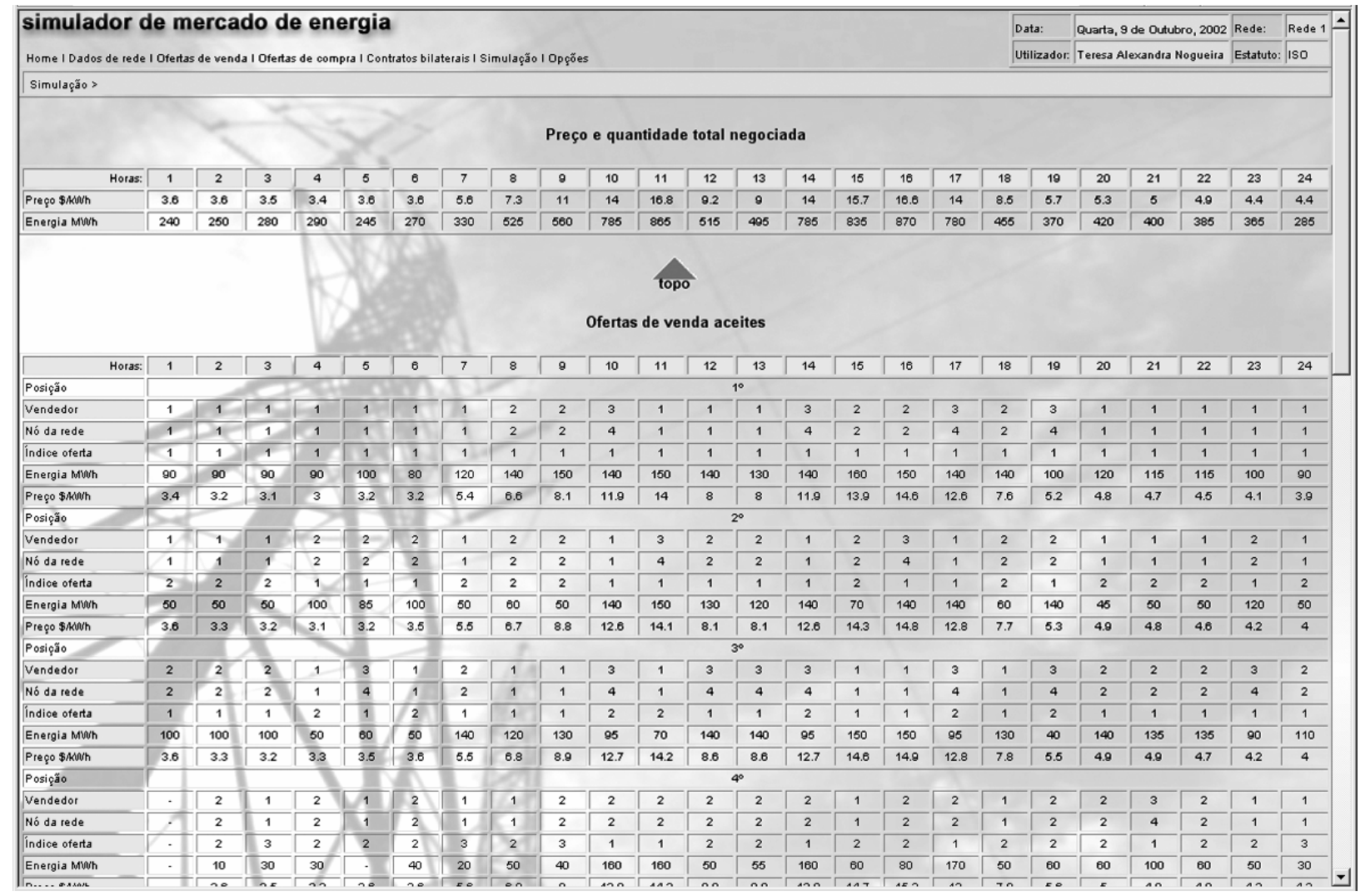

Fig. 4. View of the simulator interface: total amount of energy/price and accepted bids for the next 24 hours.

Other demand bids are quantity/price bids. The matching algorithm has cleared the market at a price of 0,073 $€ / \mathrm{MWh}$, for a total amount of energy of $525 \mathrm{MWh}$. This clearing price will be the price at which every accepted bid will be transacted. In the example illustrated by figure 2, every demand bid has been satisfied, while some seller bids have been rejected (those with prices above the clearing price of $0,073 € / \mathrm{MWh}$ ). If the demand curve should include other bids beyond the clearing point, such bids would not be satisfied by the matching algorithm. Being that the case, it is up to the ISO to decide whether to buy extra amounts of energy to meet demand or not.

The results of the matching process are published, stating the matching bids and the uniform price that has cleared the market for each hour of the next day. The market operator combines the resulting dispatch with the bilateral contracts for the next day.

Figure 4 shows the view provided by the simulator interface, which indicates the total amount of energy and price, as well as detailed information about all the accepted bids, for each of the 24 hours of the next day.

The predefined bilateral contracts details and historic data follow a typical daily load curve. Figure 5 shows the demand curves that resulted from the simulation - the curve that resulted form the market operator matching algorithm, and the total demand curve, which includes the bilateral contracts demand. Figure 6 shows the uniform price curve along the 24 hours.

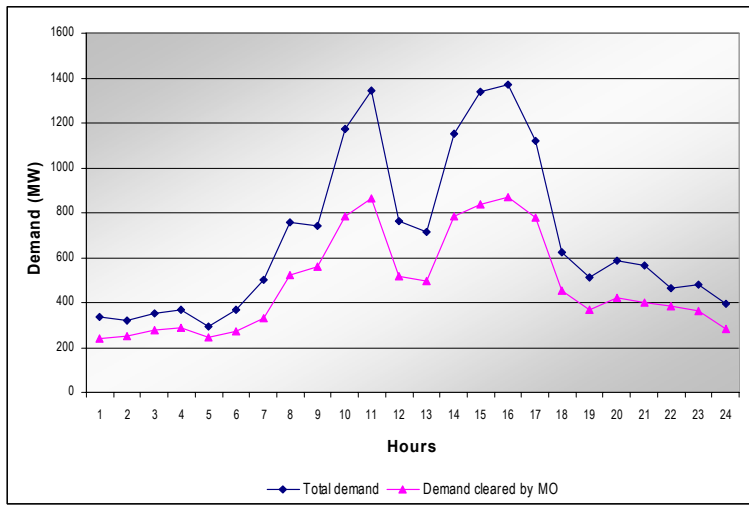

Fig. 5. Simulation results: demand curves for the next day.

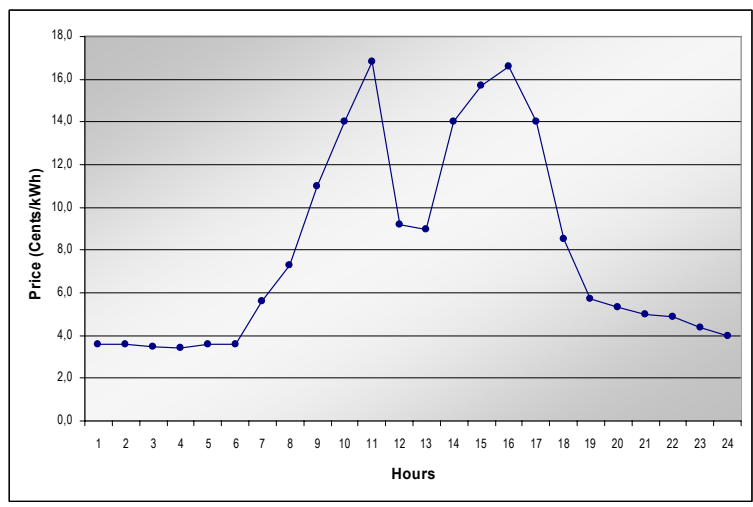

Fig. 6. Simulation results: price curve for the next day. 
The system operator then investigates the physical feasibility of the market clearing, by performing a technical validation of the contracts that have been accepted by the market operator. A DC model is used to determine whether the power lines' transmission capacity is exceeded. If that is the case, an optimal power flow algorithm finds a feasible dispatch, and the results are, again, published, listing the definitive accepted bids and what energy is to be transacted at what price.

\section{Conclusion}

In order to participate in a deregulated competitive market one must understand the new structures and roles that are put in place. A simulation model of an electricity wholesale market can be a valuable tool that provides training and deeper knowledge of the processes that are involved, namely at the economic dimension of the electricity sector. The simulation prototype that has been developed is a first approach to simulate a day-ahead market, already including some complexity in terms of the bids that are posted by participants. It will be progressively enriched with intra-daily market sessions, more complex bid conditions and flexibility. Alternative algorithms are to be included, namely an $\mathrm{AC}$ model to consider losses. The main objective is to establish a simulation tool that will predict the actual behavior of the agents in the wholesale market, providing a more realistic approach.

\section{References}

[1] R. Green, "Draining the Pool: the Reform of Electricity Trading in England and Walles," Energy Policy, Apr. 1988.

[2] M. J. Gonçalves and Z. Vale, "Market Power Analysis - Strategic Coalition Impact on Market Power", $2^{\text {nd }}$ IASTED International Conference on Power and Energy Systems, Crete, Greece, June 25-28, 2002.

[3] B. Flechner, " Business Management Systems for Energy Trading in an Open Electricity Market", EPSOM'98, Zurich, Sept. 23-25, 1998.

[4] G. B. Sheblé, Computational Auction Mechanisms for Restructured Power Industry Operation, Kluwer Academic Publishers, 1999.

[5] I. Praça, C. Ramos and Z. Vale, "Competitive Electricity Markets: Simulation to Improve Decision Making”, IEEE Power Tech, Porto, Portugal, Sept. 10-13, 2001.

[6] J. Kumar, G. Sheblé, "Auction Market Simulator for Price Based Operation", IEEE Tran. Power Syst., vol. 13, Feb. 1998.

[7] S. Stoft, Power System Economics - Designing Markets for Electricity, Wiley-IEEE, 2002. 